See discussions, stats, and author profiles for this publication at: http://www.researchgate.net/publication/234471855

\title{
Galactic clusters with associated Cepheid variables. III - NGC 1647 and SZ Tauri
}

ARTICLE in THE ASTRONOMICAL JOURNAL • OCTOBER 1992

Impact Factor: 4.05 · DOI: 10.1086/116363

CITATIONS

44
DOWNLOADS

37
VIEWS

42

1 AUTHOR:

David G. Turner

Saint Mary's University

298 PUBLICATIONS 2,211 CITATIONS

SEE PROFILE 


\title{
GALACTIC CLUSTERS WITH ASSOCIATED CEPHEID VARIABLES. III. NGC 1647 AND SZ TAURI
}

\author{
DAVID G. TURNER ${ }^{1,2}$ \\ Department of Astronomy, Saint Mary's University, Halifax, Nova Scotia, B3H 3C3, Canada \\ Received 5 June 1992
}

\begin{abstract}
New photoelectric $U B V$ photometry of 45 stars and spectroscopic observations of 24 stars are presented for NGC 1647, the cluster associated with the 3d15 Cepheid SZ Tauri. The resulting photometry, spectral classifications, and radial velocities are used with previously published photoelectric, photographic, spectroscopic, and proper motion studies of NGC 1647 for a detailed cluster analysis. The newly-obtained cluster distance modulus is $V_{0}-M_{V}=8.67 \pm 0.02$ s.e. $(d=542 \pm 4 \mathrm{pc})$, and a value of $R=A_{V} / \mathrm{E}_{B-V}=3.09 \pm 0.16$ s.e. is found to describe the dust extinction in the field. A space reddening of $\mathrm{E}_{B-V}=0.29 \pm 0.01$ is derived for SZ Tau from two B-type companions which are located east-west of the Cepheid, and its resulting luminosity as an outlying cluster member is $\left\langle M_{V}\right\rangle=-3.09 \pm 0.04$. A cluster radial velocity of $-2.3 \pm 1.6 \mathrm{~km} \mathrm{~s}^{-1}$ and cluster age of $\sim 1.9 \times 10^{8} \mathrm{yr}$ are both consistent with the likely membership of SZ Tau. It is argued from its luminosity that this Cepheid is pulsating in the first overtone mode, as is also implied by its light curve and Wesselink radius. In particular, a newly derived period-luminosity relation for cluster Cepheids of the form $\left\langle M_{V}\right\rangle=-1.15-2.94 \log P_{0}$ is consistent with the results for SZ Tau only if its observed variability is due to overtone pulsation.
\end{abstract}

\section{INTRODUCTION}

The possible membership of the $3 ! 15$ Cepheid SZ Tau in the extended corona of the nearby open cluster NGC 1647 was first suggested by Efremov (1964a, b) on the basis of considerations such as: (i) spatial coincidence, (ii) similar radial velocities for SZ Tau and bright cluster stars, and (iii) implied luminosity of the Cepheid as a cluster member. The age of the cluster inferred from its main-sequence turnoff point is also gratifyingly close to the value predicted for SZ Tau based upon its pulsational period (e.g., Tammann 1970), and the interstellar reddening of the Cepheid (e.g., Schmidt 1971) also matches the range of color excesses observed for members of NGC 1647 (Turner 1976). Although SZ Tau lies $\sim 2^{\circ}$ from the projected center of NGC 1647, marginally within the observed coronal limits of the cluster projected near the Cepheid (Kovalenko 1968), it appears otherwise to be an excellent candidate for outlying cluster membership (Turner 1984). This possibility is discussed in more detail here on the basis of new photometric and spectroscopic observations for cluster stars.

This paper is the third in a series of investigations of open clusters associated with Cepheid variables. The previously studied clusters NGC 6087 (Turner 1986, Paper I) and NGC 129 (Turner et al. 1992, Paper II) differ from NGC 1647 in having been the subject of numerous previous studies. NGC 1647 has not attracted much attention

\footnotetext{
'Visiting Astronomer, Kitt Peak National Observatory, National Optical Astronomy Observatories, which is operated by the Association of Universities for Research in Astronomy, Inc. (AURA) under cooperative agreement with the National Science Foundation.

${ }^{2}$ Guest Investigator, Dominion Astrophysical Observatory, Herzberg Institute of Astrophysics, National Research Council of Canada.
}

from photometric or spectroscopic observers, presumably because its associated Cepheid is so distant from the main body of the cluster. In addition, SZ Tau has itself been the subject of some controversy owing to the unusual nature of its light curve-which has given rise to the suggestion that it may be a Type II Cepheid (see Schaltenbrand \& Tammann 1971) -and to its exotic period changes (Szabados 1977; Trammell 1987). The Wesselink radius of SZ Tau (Gieren 1985; Moffett \& Barnes 1987b) is also unusually large for the star's pulsational period, and, combined with its sinusoidal light curve, leads one to suspect that SZ Tau may be an overtone pulsator. This possibility is discussed here in conjunction with the star's likely membership in NGC 1647.

\section{OBSERVATIONS AND DATA COMPILATIONS}

\subsection{Photoelectric Photometry}

Published photometric data for stars in NGC 1647 are not numerous, despite the cluster's relatively nearby distance of $\sim 550 \mathrm{pc}$ and its location $17^{\circ}$ below the galactic plane. To our knowledge, the only previous photoelectric $U B V$ study of the cluster is that of Hoag et al. (1961), who also presented photographic data for stars located in the cluster nucleus. Additional photographic $U B V$ and $B V$ data for cluster stars are given by Hassan (1972) and Francic (1989), respectively, this last reference being primarily a comprehensive investigation of cluster membership from proper motion data. Schmidt (1984) has also published DDO and $u v b y$ photometry for three late-type stars in NGC 1647, but none of these objects are cluster members.

A program of photoelectric $U B V$ photometry for NGC 1647 was carried out on several nights in September 1981 and August 1982 using the 0.4 and $0.9 \mathrm{~m}$ telescopes at Kitt 
TABLE 1. New photoelectric data for NGC 1647 stars.

\begin{tabular}{|c|c|c|c|c|c|}
\hline CDS & Hoag & V & $\mathrm{B}-\mathrm{V}$ & U-B & $\mathrm{n}$ \\
\hline 45 & .. & 8.88 & +0.28 & -0.11 & 3 \\
\hline 22 & 2 & 9.08 & +0.20 & -0.11 & 2 \\
\hline 44 & 29 & 9.25 & +0.25 & -0.04 & 3 \\
\hline 3 & 31 & 9.62 & +0.32 & -0.06 & 1 \\
\hline 94 & 5 & 9.69 & +0.23 & -0.15 & 1 \\
\hline 42 & 30 & 9.70 & +0.33 & +0.22 & 1 \\
\hline 51 & $\ldots$ & 10.03 & +0.39 & +0.09 & 2 \\
\hline 54 & 7 & 10.10 & +0.36 & +0.00 & 2 \\
\hline 37 & 34 & 10.16 & +0.30 & +0.13 & 1 \\
\hline 10 & $\ldots$ & 10.25 & +0.48 & +0.08 & 1 \\
\hline 55 & $\ldots$ & 10.31 & +0.43 & +0.03 & 1 \\
\hline 39 & 8 & 10.32 & +0.93 & +0.48 & 1 \\
\hline 29 & 9 & 10.70 & +0.31 & +0.17 & 2 \\
\hline 13 & 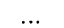 & 10.82 & +0.54 & +0.44 & 1 \\
\hline 4 & 38 & 10.92 & +0.35 & +0.31 & 1 \\
\hline 30 & 10 & 11.00 & +0.40 & +0.34 & 2 \\
\hline $40^{\mathrm{a}}$ & 11 & 11.14 & +0.87 & +0.39 & 1 \\
\hline 41 & 39 & 11.17 & +0.40 & +0.27 & 1 \\
\hline 224 & 40 & 11.22 & +0.40 & +0.34 & 1 \\
\hline 28 & 12 & 11.37 & +0.50 & +0.35 & 2 \\
\hline 52 & $\ldots$ & 11.42 & +0.93 & +0.48 & 2 \\
\hline 53 & $\ldots$ & 11.48 & +0.49 & +0.42 & 2 \\
\hline 9 & 13 & 11.59 & +0.62 & +0.48 & 2 \\
\hline 93 & 14 & 11.71 & +0.42 & +0.35 & 1 \\
\hline 46 & 43 & 11.72 & +0.51 & +0.39 & 3 \\
\hline 97 & 15 & 11.78 & +0.52 & +0.44 & 1 \\
\hline 11 & 47 & 11.94 & +0.66 & +0.46 & 1 \\
\hline 351 & $\ldots$ & 12.16 & +0.60 & +0.47 & 1 \\
\hline 98 & 16 & 12.42 & +0.88 & +0.44 & 1 \\
\hline 350 & $\ldots$ & 12.54 & +0.67 & +0.50 & 1 \\
\hline 96 & 17 & 12.65 & +0.66 & +0.49 & 1 \\
\hline 178 & 18 & 12.74 & +0.77 & +0.31 & 2 \\
\hline 179 & 19 & 12.79 & +0.66 & +0.49 & 2 \\
\hline 243 & 58 & 13.15 & +0.93 & +0.40 & 2 \\
\hline $51 \mathrm{~s}^{\mathrm{b}}$ & $\ldots$ & 13.20 & +0.75 & +0.35 & 1 \\
\hline 256 & $\ldots$ & 13.37 & +0.75 & +0.24 & 1 \\
\hline 95 & 65 & 13.55 & +0.79 & +0.23 & 1 \\
\hline 214 & 20 & 13.64 & +0.93 & +0.27 & 2 \\
\hline 249 & 21 & 14.01 & +0.79 & +0.30 & 2 \\
\hline 242 & 22 & 14.09 & +0.83 & +0.34 & 2 \\
\hline 173 & 23 & 14.11 & +0.87 & +0.32 & 2 \\
\hline 358 & 24 & 14.47 & +0.99 & +0.31 & 1 \\
\hline 426 & 25 & 15.21 & +1.48 & +1.43 & 1 \\
\hline 427 & 26 & 15.58 & +0.88 & +0.99 & 1 \\
\hline 428 & 27 & 15.64 & +1.05 & +1.32 : & 2 \\
\hline
\end{tabular}

a Close double. Both stars in diaphragm

b South of CDS 51 .

Peak National Observatory; roughly $70 \%$ of the observations, and all of those for faint program stars, were obtained with the larger telescope. The photomultiplier and filter sets for these observing runs were similar to those described in Paper II, and, as noted there, an effort was made to investigate the internal consistency of the resulting data to assure that they were tied closely to the $U B V$ system of Johnson \& Morgan (1953). Small systematic effects were found in the color data published earlier by Turner (1984) for stars in the immediate vicinity of SZ Tau, and these are corrected here. The primary source of star numbering in the cluster, as adopted by Mermilliod (1976a) and the Stellar Data Center (CDS) at Strasbourg, is an extension of that used in the survey by Cuffey (1937). We have followed this scheme in the present study.

The photometric data are presented in columns 3-5 of Table 1, with star identifications from the CDS and Hoag et al. (1961, see description of numbering scheme adopted in Paper II) given in columns 1 and 2, respectively. The number of individual nights of observation for each star is listed in column 6. Relative uncertainties in magnitudes and colors are similar to those cited in Paper II, and the data are an excellent match to the photoelectric photometry of Hoag et al. for the 23 stars in common. The mean differences in $V, B-V$, and $U-B$ (This paper-Hoag et al. ) are $+0.004 \pm 0.006$ s.e., $-0.002 \pm 0.005$ s.e., and $0.004 \pm 0.012$ s.e., respectively, excluding CDS 249 (for which the single observation by Hoag et al. is suspect) and CDS 427 (for which our single observation is suspect). A combination of the photoelectric data from Hoag et al., Turner (1984, corrected), and this paper is given in Table 2. We have assumed for this purpose that the Hoag et al. data are all single observations, as determined from the remarks in the U.S. Naval Observatory Catalogue. Cluster membership probabilities from Francic (1989) are included along with the photometric data.

In previous papers of this series we have commented upon the need for additional photoelectric observations of Cepheid cluster stars, particularly faint cluster members. The situation for NGC 1647 is less critical in this regard owing to the relative proximity of the cluster. Its mainsequence is not expected to be populated to stellar magnitudes much fainter than $V \approx 14$, which is fortunate given the fact that the accuracy of the photoelectric data in Table 2 is suspect for stars with $V>15$. As a precautionary measure, we restricted our analysis to stars with $V<15$.

\subsection{Photographic Data}

A large sample of cluster stars is still desirable for a thorough study of NGC 1647. Because of this, we have included in our analysis an extensive selection of stars observed only by means of photographic photometry. The $U B V$ data of Hoag et al. (1961) and Hassan (1972) were carefully examined and adjusted to the system of Table 2 for this purpose, and combined with the $B V$ data of Francic (1989) where possible. The photographic data appear to have rather sizable associated uncertainties for $V>14.5$, so we have generally omitted such stars from this compilation. The combined data for the stars in the resulting sample are presented in Table 3 along with the associated cluster membership probabilities from Francic (1989).

\subsection{Spectroscopic Data}

The brightness of cluster stars makes NGC 1647 an attractive object for spectroscopic observations, yet most previous studies of this type (Zug 1933; Svolopoulos 1962; Hoag \& Applequist 1965) have been for the sole purpose of spectral classification. We undertook a more ambitious spectroscopic program for NGC 1647 using the Cassegrain spectrograph on the $1.8 \mathrm{~m}$ telescope of the Dominion Astrophysical Observatory during 10 nights in October 1984, September 1985, October 1990, and September/October 1991. The 1984/85 observations were photographic imageintensifier spectra at a dispersion of $16 \AA \mathrm{mm}^{-1}$, while the $1990 / 91$ observations were CCD spectra at a dispersion of $60 \AA \mathrm{mm}^{-1}$ (see Paper II) with mean signal-to-noise ratios ranging between 130 and 400 . Both sets of spectra were centered in the blue spectral region around $4000 \AA$. 
TABLE 2. Combined photoelectric data for NGC 1647 stars.

\begin{tabular}{|c|c|c|c|c|c|c|c|c|c|c|c|c|c|}
\hline CDS/HD & Hoag & V & B-V & U-B & $\mathrm{n}$ & MP & CDS & Hoag & V & $B-V$ & U-B & $\mathrm{n}$ & MP \\
\hline 29023 & $\ldots$ & 8.07 & +0.20 & -0.16 & 2 &.$^{a}$ & 53 & $\ldots$ & 11.48 & +0.49 & +0.42 & 2 & 1.00 \\
\hline 29181 & $\ldots$ & 8.39 & +0.28 & +0.12 & 2 & $\ldots^{b}$ & 9 & 13 & 11.60 & +0.60 & +0.48 & 3 & $1.00^{\mathrm{b}}$ \\
\hline 29403 & $\ldots$ & 8.51 & +0.21 & -0.12 & 2 & $\ldots^{a}$ & 93 & 14 & 11.71 & +0.42 & +0.35 & 2 & 1.00 \\
\hline 15 & 1 & 8.61 & +0.36 & -0.05 & 1 & 1.00 & 46 & 43 & 11.72 & +0.51 & +0.39 & 3 & 1.00 \\
\hline 45 & $\ldots$ & 8.88 & +0.28 & -0.11 & 3 & 1.00 & 97 & 15 & 11.79 & +0.53 & +0.44 & 2 & 1.00 \\
\hline 22 & 2 & 9.08 & +0.20 & -0.10 & 3 & 1.00 & 11 & 47 & 11.94 & +0.66 & +0.46 & 1 & 0.98 \\
\hline 44 & 29 & 9.25 & +0.25 & -0.04 & 3 & 1.00 & SZ-1 & $\ldots$ & 12.09 & +2.06 & +1.46 & 1 & $\ldots{ }^{b}$ \\
\hline 102 & 3 & 9.34 & +0.32 & +0.01 & 1 & 1.00 & 351 & $\ldots$ & 12.16 & +0.60 & +0.47 & 1 & 1.00 \\
\hline 16 & 4 & 9.34 & +0.90 & +0.57 & 1 & 0.00 & 98 & 16 & 12.44 & +0.89 & +0.44 & 2 & 0.00 \\
\hline 29402 & $\cdots$ & 9.35 & +0.29 & +0.26 & 2 & $\ldots^{b}$ & 350 & $\cdots$ & 12.54 & +0.67 & +0.50 & 1 & 1.00 \\
\hline 3 & 31 & 9.62 & +0.32 & -0.06 & 1 & 1.00 & 96 & 17 & 12.66 & +0.67 & +0.49 & 2 & 1.00 \\
\hline 94 & 5 & 9.69 & +0.23 & -0.16 & 2 & 1.00 & 178 & 18 & 12.74 & +0.76 & +0.31 & 3 & 0.00 \\
\hline 42 & 30 & 9.70 & +0.33 & +0.22 & 1 & 1.00 & 179 & 19 & 12.80 & +0.66 & +0.49 & 3 & $0.00^{c}$ \\
\hline 51 & $\ldots$ & 10.03 & +0.39 & +0.09 & 2 & 1.00 & 243 & 58 & 13.15 & +0.93 & +0.40 & 2 & 0.00 \\
\hline 29527 & $\ldots$ & 10.06 & +0.33 & +0.32 & 2 & $\ldots b$ & $51 \mathrm{~s}$ & $\ldots$ & 13.20 & +0.75 & +0.35 & 1 & $\ldots^{a}$ \\
\hline 99 & 6 & 10.09 & +0.41 & +0.12 & 1 & 1.00 & 256 & $\ldots$ & 13.37 & +0.75 & +0.24 & 1 & 1.00 \\
\hline 54 & 7 & 10.10 & +0.36 & +0.00 & 3 & 1.00 & 95 & 65 & 13.55 & +0.79 & +0.23 & 1 & 1.00 \\
\hline 37 & 34 & 10.16 & +0.30 & +0.13 & 1 & 1.00 & 214 & 20 & 13.63 & +0.93 & +0.28 & 3 & 0.97 \\
\hline 10 & $\ldots$ & 10.25 & +0.48 & +0.08 & 1 & 1.00 & 249 & 21 & 14.01 & +0.80 & +0.29 & 3 & 0.98 \\
\hline 55 & $\cdots$ & 10.31 & +0.43 & +0.03 & 1 & 1.00 & 242 & 22 & 14.10 & +0.84 & $+0.31:$ & 3 & $0.98^{c}$ \\
\hline 39 & 8 & 10.33 & +0.91 & +0.47 & 2 & 0.00 & 173 & 23 & 14.11 & +0.88 & +0.31 & 3 & 0.00 \\
\hline 29 & 9 & 10.70 & +0.31 & +0.17 & 3 & 1.00 & SZ-2 & $\ldots$ & 14.21 & +0.83 & +0.64 & 1 & $\ldots^{b}$ \\
\hline 13 & $\ldots$ & 10.82 & +0.54 & +0.44 & 1 & $1.00^{\mathrm{b}}$ & 358 & 24 & 14.46 & +0.98 & $+0.41:$ & 2 & 0.97 \\
\hline 4 & 38 & 10.92 & +0.35 & +0.31 & 1 & 1.00 & 426 & 25 & 15.19 & +1.49 & +1.43 & 2 & $\ldots$ \\
\hline 30 & 10 & 11.00 & +0.40 & +0.34 & 3 & 0.00 & 427 & 26 & 15.40: & +1.03: & +0.99 & 2 & $\ldots$ \\
\hline 40 & 11 & 11.13 & +0.86 & +0.40 & 2 & 0.00 & 428 & 27 & 15.61: & +1.05 & $+1.32:$ & 3 & $\ldots$ \\
\hline 41 & 39 & 11.17 & +0.40 & +0.27 & 1 & 1.00 & 429 & 28 & 16.11 & +0.88 & $\ldots$ & 1 & $\ldots$ \\
\hline 224 & 40 & 11.22 & +0.40 & +0.34 & 1 & 1.00 & & & & & & & \\
\hline 28 & 12 & 11.37 & +0.51 & +0.35 & 3 & 1.00 & & & & & & & \\
\hline 52 & $\ldots$ & 11.42 & +0.93 & +0.48 & 2 & 0.00 & & & & & & & \\
\hline
\end{tabular}

a Probable cluster member.

b Probable non-member of cluster.

c Likely background star.

The image-intensifier spectra were scanned and measured for radial velocity using the PDS microdensitometer at the David Dunlap Observatory, University of Toronto, while the CCD spectra were processed using the IRAF software package and reduced for radial velocity and rotational velocity measurement as in Paper II using the software routines REDUCE and vCROSS developed by Graham Hill (Hill 1982; Hill et al. 1982; Hill \& Fisher 1986). The CCD spectra were also suitable for reliable spectral classification due to their high signal-to-noise ratios (see Paper II). The image-intensifier spectra encompass a smaller spectral region which does not include all of the spectral lines appropriate for standard MK spectral classification. However, the plates do cover the region of $\mathrm{Ca} \mathrm{K}$ and a few lines of $\mathrm{He} \mathrm{I}$, in addition to the lines of higher Balmer series members, and it was possible to use these features in an intercomparison of the spectra to derive reasonably accurate classifications for the observed stars. All are of late B and early $A$ spectral type. It was also possible to infer luminosity classes and degrees of rotational broadening from the spectra by using the hydrogen line wings and the widths of the $\mathrm{He} \mathrm{I}$ lines, respectively. The resulting spectral classification information for cluster stars is summarized in Table 4. Table 5 presents the new radial velocity data.

Unlike previous spectroscopic surveys of NGC 1647, the present program was restricted to the early-type mem- bers of the cluster. The late-type stars in NGC 1647 are all field stars according to the available proper motion (Francic 1989) and radial velocity (Glushkova \& Rastorguev 1991) data. Of the 24 stars in Table 5, 20 have proper motion membership probabilities of 1.00 (Francic 1989), although one of these, CDS 13, seems likely to be a nonmember on photometric grounds. The three HD stars near SZ Tau lack information of this type; however, the AGK3 proper motions for HD 29023 and HD 29403 are consistent with outlying cluster membership. Of the remaining two stars, HD 29527 lacks proper motion data, and CDS 30 is not a cluster member according to Francic. Both stars are only marginal candidates for cluster membership according to the photometric data, and foreground status seems very likely. Their velocities are also slightly more positive than those of proper motion members, which is consistent with their nonmembership status. The mean velocity for the 21 recognized members of NGC 1647 is $-2.3 \pm 1.6$ s.e. $\mathrm{km} \mathrm{s}^{-1}$. It is not possible to properly address the effects of velocity variability on this sample with so few observations, but it does seem unlikely to affect the cluster mean velocity by more than $1-2 \mathrm{~km} \mathrm{~s}^{-1}$.

The most extensive radial velocity measurements for SZ Tau are those published by Haynes (1914), Gieren (1985), and Barnes et al. (1987), which are based upon the analysis of photographic spectra, CCD spectra, and 
TABLE 3. Combined photographic data for NGC 1647 stars.

\begin{tabular}{|c|c|c|c|c|c|c|c|c|c|c|c|}
\hline CDS & Hoag & V & B-V & U-B & MP & $\mathrm{CDS}$ & Hoag & V & $B-V$ & U-B & MP \\
\hline 49 & 32 & 9.99 & +0.41 & +0.20 & 1.00 & 223 & 68 & 13.58 & +0.86 & +0.41 & 0.99 \\
\hline 50 & 33 & 10.13 & +0.21 & -0.18 & 1.00 & 225 & 72 & 13.64 & +1.30 & +1.11 & 0.00 \\
\hline 5 & 35 & 10.20 & +0.24 & -0.09 & 1.00 & 174 & 69 & 13.66 & +1.17 & +1.04 & 0.00 \\
\hline 31 & 37 & 10.36 & +0.28 & +0.04 & 1.00 & 254 & 71 & 13.71 & +0.81 & $+0.45:$ & $\ldots{ }^{c}$ \\
\hline 48 & 36 & 10.39 & +0.34 & +0.07 & 1.00 & 188 & 70 & 13.74 & +1.03 & $+0.52:$ & $0.99^{b}$ \\
\hline 14 & 41 & 11.43 & +0.51 & +0.35 & 1.00 & 258 & 73 & 13.75 & +0.93 & +0.45 & 0.00 \\
\hline 226 & $\ldots$ & 11.46 & +1.92 & +1.95 & 0.00 & 221 & 74 & 13.76 & +1.04 & +0.58 & 0.00 \\
\hline 33 & 42 & 11.47 & +0.38 & $+0.37:$ & 1.00 & 215 & 76 & 13.85 & +0.91 & +0.34 & 0.99 \\
\hline 35 & 45 & 11.74 & +0.41 & $+0.35:$ & 1.00 & 416 & 75 & 13.86 & +0.83 & +0.35 & $0.00^{\mathrm{c}}$ \\
\hline 176 & 44 & 11.79 & +0.47 & +0.30 & 1.00 & 204 & 77 & 13.88 & +1.00 & +0.51 & 0.99 \\
\hline 6 & 48 & 12.18 & +1.44 & +1.69 & 0.00 & 255 & 78 & 13.95 & +0.85 & +0.42 & $0.97^{\mathrm{c}}$ \\
\hline 38 & 46 & 12.28 & +0.61 & +0.36 & 1.00 & 222 & 79 & 13.95 & +0.96 & +0.44 & 0.96 \\
\hline 47 & 49 & 12.40 & +0.75 & +0.41 & 1.00 & 359 & 80 & 13.99 & +1.10 & +0.63 & $0.99^{b}$ \\
\hline 7 & 51 & 12.41 & +0.80 & +0.27 & $1.00^{\mathrm{b}}$ & 190 & 81 & 14.16 & +0.83 & +0.37 & $0.98^{c}$ \\
\hline 92 & 50 & 12.42 & +1.22 & +0.98 & 0.00 & 208 & 82 & 14.32 & +1.03 & +0.68 & 0.00 \\
\hline 210 & 52 & 12.45 & +0.61 & +0.31 & 1.00 & 440 & $\ldots$ & 14.36 & +1.16 & +0.82 & 0.98 \\
\hline 8 & 53 & 12.52 & +0.64 & +0.39 & 1.00 & 353 & 83 & 14.38 & +0.95 & +0.48 & 0.98 \\
\hline 36 & 54 & 12.62 & +0.96 & +0.69 & 0.00 & 251 & 86 & 14.42 & +1.45 & +0.93 & 0.93 \\
\hline 177 & 56 & 12.88 & +0.70 & $+0.22:$ & 1.00 & 357 & 84 & 14.43 & +1.01 & +0.27 & 0.00 \\
\hline 32 & 55 & 12.89 & +0.69 & +0.24 & 1.00 & 213 & 85 & 14.46 & +1.15 & +0.73 & 0.00 \\
\hline 356 & $\cdots$ & 12.98 & +1.86 & +1.96 & 0.00 & 354 & 87 & 14.51 & +0.73 & +0.26 & $0.40^{\mathrm{c}}$ \\
\hline 43 & 57 & 13.11 & +0.78 & +0.45 & 0.99 & 201 & 90 & 14.54 & +0.98 & +0.47 & 0.98 \\
\hline 202 & 59 & 13.12 & +0.78 & +0.41 & 1.00 & 430 & 88 & 14.54 & +1.02 & +0.52 & $\ldots^{\mathrm{a}}$ \\
\hline 237 & 62 & 13.15 & +0.76 & +0.37 & 0.99 & 218 & 92 & 14.56 & +1.12 & +0.58 & 0.00 \\
\hline 212 & 60 & 13.20 & +0.80 & +0.32 & 1.00 & 360 & 89 & 14.58 & +0.96 & +0.49 & 0.98 \\
\hline 211 & 61 & 13.21 & +0.76 & +0.32 & 1.00 & 355 & $\ldots$ & 14.60 & +0.99 & +0.80 & 0.98 \\
\hline 238 & 63 & 13.27 & +1.28 & $+1.39:$ & 0.00 & 361 & 91 & 14.61 & +1.20 & $+0.64:$ & 0.98 \\
\hline 205 & 64 & 13.31 & +0.75 & +0.25 & 1.00 & 220 & 96 & 14.63 & +1.03 & $+0.68:$ & 0.07 \\
\hline 217 & 66 & 13.36 & +0.91 & +0.64 & 1.00 & 207 & 95 & 14.66 & +1.05 & +0.37 & 0.98 \\
\hline 250 & 67 & 13.41 & +0.84 & +0.40 & 0.00 & & & & & & \\
\hline
\end{tabular}

a Probable cluster member.

b Probable non-member of cluster.

c Likely background star.

radial velocity scanner data, respectively. Four additional short series of radial velocity measurements for SZ Tau available in the literature are listed by Szabados (1991). The proper phase matching of these velocities is made difficult by the rather exotic period variability observed for this Cepheid (Szabados 1977; Trammell 1987), but can be accomplished in a fairly reliable fashion. The velocity data published by Gieren exhibit smaller internal scatter than those of Haynes and Barnes et al., but they are $4.1 \mathrm{~km} \mathrm{~s}^{-1}$ more positive than the latter (Moffet \& Barnes 1987a). This discrepancy may be either an instrumental effect or evidence for duplicity (see Szabados 1988a, 1991). Gieren (1985) has demonstrated that there is no evidence for a hot companion to SZ Tau in the star's photometric data, and this is confirmed by spectroscopic observations in the ultraviolet (Böhm-Vitense \& Proffitt 1985) and for the Ca II K line (Evans 1985). The possibility that the Cepheid is a spectroscopic binary, however, has not been ruled out. Additional observations would seem to be essential for a proper investigation of this possibility, as noted by Szabados (1991).

If Gieren's radial velocity measurements for SZ Tau are adjusted to the system defined by the measurements of Haynes and Barnes et al., the systemic velocity for the Cepheid is found to be $-3.5 \pm 1.0 \mathrm{~km} \mathrm{~s}^{-1}$ (our estimate). Gieren (1985) derived a value of $+0.5 \pm 0.85$ (s.e.) $\mathrm{km} \mathrm{s}^{-1}$ from his measurements, while Caldwell \& Coulson (1987) obtain a value of $+0.3 \mathrm{~km} \mathrm{~s}^{-1}$ with the same data. The various estimates for the systemic velocity of the Cepheid cited by Szabados (1991) yield a mean velocity for SZ Tau of $-1.4 \pm 0.8 \mathrm{~km} \mathrm{~s}^{-1}$, which is in excellent agreement with the value of $-2.3 \pm 1.6 \mathrm{~km} \mathrm{~s}^{-1}$ found here for NGC 1647. Combined with the Cepheid's proper motion from the AGK3, the space velocity data present a strong case for cluster membership of the Cepheid. Foreground status would seem to require a velocity for the Cepheid $\sim 6$ $\mathrm{km} \mathrm{s}^{-1}$ more positive than the observed value, which is outside the range of the various estimates cited by Szabados. The membership of SZ Tau in NGC 1647 has never been questioned previously in the literature. However, its spatial location at the outer edge of the cluster corona makes it imperative to use tests such as these to demonstrate its likely cluster membership in convincing fashion.

\section{ANALYSIS}

\subsection{Interstellar Reddening}

The color excesses of cluster stars deduced from their spectral classifications and observed colors are presented in Fig. 1. The stellar sample used here was restricted to stars having classifications from this paper or Turner (1984), and gives a fairly clear picture of the slope of the reddening 
TABLE 4. Spectral classification information for NGC 1647 stars.

\begin{tabular}{|c|c|c|c|c|c|c|c|}
\hline CDS/HD & Hoag & Spectral Type & Source & $\mathrm{CDS} / \mathrm{HD}$ & Hoag & Spectral Type & Source \\
\hline 15 & 1 & $\begin{array}{l}\text { B7 } \\
\text { B8 III } \\
\text { B7 Ve } \\
\text { B7.5 Illev }\end{array}$ & $\begin{array}{r}1 \\
2 \\
3 \\
5,6\end{array}$ & 22 & 2 & $\begin{array}{l}\text { B8 } \\
\text { B9 II } \\
\text { B8 III } \\
\text { B8 IVn }\end{array}$ & $\begin{array}{l}1 \\
2 \\
3 \\
6\end{array}$ \\
\hline 102 & 3 & $\begin{array}{l}\text { B8 } \\
\text { B9 III } \\
\text { B8 IVnn }\end{array}$ & $\begin{array}{l}1 \\
2 \\
6\end{array}$ & 94 & 5 & $\begin{array}{l}\text { A0 } \\
\text { B9 III } \\
\text { B7 Vn }\end{array}$ & $\begin{array}{l}1 \\
2 \\
5\end{array}$ \\
\hline 29023 & $\ldots$ & B7.5 III & 6 & 29181 & $\ldots$ & A7 V & 4 \\
\hline 29403 & $\ldots$ & B8 III & 6 & 16 & 4 & $\mathrm{~K} 0 \mathrm{~V}$ & 2 \\
\hline 45 & $\ldots$ & $\begin{array}{l}\text { B9 } \\
\text { B7.5 IVn }\end{array}$ & $\begin{array}{l}1 \\
6\end{array}$ & 44 & 29 & $\begin{array}{l}\text { B9 } \\
\text { B8 IVnn }\end{array}$ & $\begin{array}{l}1 \\
6\end{array}$ \\
\hline 29402 & $\ldots$ & $\mathrm{A} 2 \mathrm{~V}$ & 4 & 49 & 32 & $\mathrm{~A} 0$ & 1 \\
\hline 3 & 31 & $\begin{array}{l}\text { B9 } \\
\text { B7.5 IV }\end{array}$ & $\begin{array}{l}1 \\
5\end{array}$ & 42 & 30 & $\begin{array}{l}\mathrm{A} 0 \\
\mathrm{~B} 9.5 \mathrm{IV}\end{array}$ & $\begin{array}{l}1 \\
5\end{array}$ \\
\hline 51 & $\ldots$ & $\begin{array}{l}\text { A0 } \\
\text { B9 IVnn }\end{array}$ & $\begin{array}{l}1 \\
5\end{array}$ & 37 & 34 & $\begin{array}{l}\mathrm{A} 0 \\
\mathrm{~B} 9.5 \mathrm{IV}\end{array}$ & $\begin{array}{l}1 \\
5\end{array}$ \\
\hline 29527 & $\ldots$ & $\mathrm{A} 2 \mathrm{~V}$ & 6 & 50 & 33 & B7 & 1 \\
\hline 99 & 6 & $\begin{array}{l}\text { B9 } \\
\text { B9 IV } \\
\text { B9 IVn }\end{array}$ & $\begin{array}{l}1 \\
2 \\
5\end{array}$ & 54 & 7 & $\begin{array}{l}\text { B8 } \\
\text { A0 V } \\
\text { B8 IVn }\end{array}$ & $\begin{array}{l}1 \\
3 \\
5\end{array}$ \\
\hline 5 & 35 & B7 & 1 & 31 & 37 & A2 & 1 \\
\hline 10 & $\ldots$ & $\begin{array}{l}\text { B9 } \\
\text { B7.5: IV }\end{array}$ & $\begin{array}{l}1 \\
5\end{array}$ & 55 & $\ldots$ & $\begin{array}{l}\text { B9 } \\
\text { B7 V }\end{array}$ & $\begin{array}{l}1 \\
5\end{array}$ \\
\hline 39 & 8 & $\begin{array}{l}\text { G0 } \\
\text { G8 III }\end{array}$ & $\begin{array}{l}1 \\
2\end{array}$ & 13 & $\ldots$ & $\begin{array}{l}\text { A3 } \\
\text { A3 V }\end{array}$ & $\begin{array}{l}1 \\
5\end{array}$ \\
\hline 48 & 36 & $\mathrm{~A} 0$ & 1 & 40 & 11 & G3 & 1 \\
\hline 29 & 9 & $\begin{array}{l}\mathrm{A} 2 \\
\mathrm{~A} 0 \mathrm{IV} \\
\mathrm{B} 9.5 \mathrm{Vn}\end{array}$ & $\begin{array}{l}1 \\
2 \\
5\end{array}$ & 4 & 38 & $\begin{array}{l}\text { Al } \\
\text { A0 Vn }\end{array}$ & $\begin{array}{l}1 \\
5\end{array}$ \\
\hline 30 & 10 & $\begin{array}{l}\mathrm{A}^{2} \\
\mathrm{~A} 2 \mathrm{~V}\end{array}$ & $\begin{array}{l}1 \\
5\end{array}$ & 41 & 39 & $\begin{array}{l}\mathrm{A} 2 \\
\mathrm{~B} 9.5 \mathrm{~V}\end{array}$ & $\begin{array}{l}1 \\
5\end{array}$ \\
\hline 224 & 40 & $\begin{array}{l}\mathrm{A} 0 \\
\mathrm{~A} 0 \mathrm{~V}\end{array}$ & $\begin{array}{l}1 \\
5\end{array}$ & 28 & 12 & $\begin{array}{l}\mathrm{A} 3 \\
\mathrm{~A} 3 \mathrm{Vn}\end{array}$ & $\begin{array}{l}1 \\
5\end{array}$ \\
\hline 53 & $\ldots$ & A5 & 1 & 9 & 13 & A3 & 1 \\
\hline 93 & 14 & A4 & 1 & 46 & 43 & $\mathrm{~A} 2$ & 1 \\
\hline 210 & 52 & A7 & 1 & & & & \\
\hline
\end{tabular}

Sources: 1. Zug (1933), 2. Svolopoulos (1962), 3. Hoag \& Applequist (1965), 4. Turner (1984), 5. This paper (photographic spectra), 6. This paper (CCD spectra).

law for the NGC 1647 field. A regression analysis yields $\mathrm{E}_{U-B} / \mathrm{E}_{B-V}=0.76 \pm 0.02$ s.e. for the sample of 26 stars, and $0.77 \pm 0.04$ s.e. if the data for HD 29181 and CDS 28 are excluded. Both of these stars are mid-to-late A-type stars, where stellar rotation affects the expected intrinsic colors, and both depart from the main trend in Fig. 1. If the analysis is further restricted to the eight stars with spectral classifications based on high signal-to-noise ratio CCD spectra, the resulting reddening slope is $0.77 \pm 0.02$ s.e., identical to the second value derived above as well as to the value found for this region of the Galaxy in the survey by Turner (1976). A reddening slope of $\mathrm{E}_{U-B} /$ $E_{B-V}=0.77$ seems to be the preferred result for this field, and is consistent with the nature of the reddening expected for dust clouds belonging to this portion of Gould's Belt, as noted by Turner (1989). This value was therefore adopted for the dereddening of cluster B, A, and F-type stars. Steeper reddening slopes are expected for late-type cluster stars due to bandwidth effects on their $U B V$ colors (e.g., Fernie 1963), and we have consequently used reddening slopes for these program stars which follow the colordependent trends predicted theoretically by Buser (1978) for stars of late spectral type.

Figure 2 is the color-color diagram for cluster stars based upon the data of Tables 2 and 3. Spectral types are available for most of the bright stars, and it appears that the cluster field is devoid of unreddened B-, A-, and F-type stars, with a minimum reddening of roughly $\mathrm{E}_{B-V}=0.30$ applying to all likely cluster members. The combined information on proper motion membership probabilities, spectral types, and radial velocities (for the brighter stars) proves to be an effective mixture for the analysis of the 
TABLE 5. New radial velocity data for NGC 1647 stars.

\begin{tabular}{|c|c|c|c|c|}
\hline $\mathrm{CDS} / \mathrm{HD}$ & HJD $2440000+$ & $\begin{array}{l}\mathrm{V}_{\mathrm{R}} \\
\mathrm{km} \mathrm{s}^{-1}\end{array}$ & $\begin{array}{c}\text { Adopted } V_{R} \\
\mathrm{~km} \mathrm{~s}^{-1}\end{array}$ & $\begin{array}{l}\mathrm{V} \sin \mathrm{i} \\
\mathrm{km} \mathrm{s}^{-1}\end{array}$ \\
\hline 29023 & 8515.0209 & $-5 \pm 7$ & -5 & $140 \pm 14$ \\
\hline 29403 & $\begin{array}{l}6328.0212 \\
8515.0482\end{array}$ & $\begin{array}{r}-3 \pm 3 \\
+13 \pm 8\end{array}$ & -1 & $137 \pm 15$ \\
\hline 15 & $\begin{array}{l}6004.9614 \\
8180.8691\end{array}$ & $\begin{array}{r}-1 \pm 8 \\
-16 \pm 7\end{array}$ & -9 & $72 \pm 17$ \\
\hline 45 & $\begin{array}{l}6004.9722 \\
8180.9417\end{array}$ & $\begin{array}{l}+16 \pm 2 \\
+11 \pm 7\end{array}$ & +16 & $250 \pm 4$ \\
\hline 22 & $\begin{array}{l}6005.0111 \\
8541.0443\end{array}$ & $\begin{array}{r}-6 \pm 1 \\
-11 \pm 6\end{array}$ & -6 & $285 \pm 7$ \\
\hline 44 & $\begin{array}{l}6004.9864 \\
8542.9846\end{array}$ & $\begin{array}{l}-3 \pm 15 \\
-3 \pm 6\end{array}$ & -3 & $283 \pm 15$ \\
\hline 102 & $\begin{array}{l}6004.9975 \\
8544.9594\end{array}$ & $\begin{array}{l}-19 \pm 1 \\
-19 \pm 6\end{array}$ & -19 & $298 \pm 10$ \\
\hline 3 & 6329.0295 & $-4 \pm 9$ & -4 & \\
\hline 94 & 6005.0534 & $-4 \pm 7$ & -4 & \\
\hline 42 & 6331.9457 & $-5 \pm 1$ & -5 & \\
\hline 51 & 6331.9308 & $+1 \pm 1$ & +1 & \\
\hline 29527 & $\begin{array}{l}6328.0309 \\
8515.0853\end{array}$ & $\begin{array}{l}+5 \pm 1 \\
+5 \pm 6\end{array}$ & $+5^{a}$ & $165 \pm 20$ \\
\hline 99 & 6331.9440 & $-7 \pm 7$ & -7 & \\
\hline 54 & 6331.9371 & $+0 \pm 7$ & +0 & \\
\hline 37 & 6329.0218 & $+4 \pm 6$ & +4 & \\
\hline 10 & 6329.0107 & $+7 \pm 6$ & +7 & \\
\hline 55 & 6327.0369 & $+0 \pm 4$ & +0 & \\
\hline 29 & 6326.9713 & $+1 \pm 3$ & +1 & \\
\hline 13 & 6327.0286 & $-0 \pm 7$ & $-0^{a}$ & \\
\hline 4 & 6327.0157 & $+2 \pm 5$ & +2 & \\
\hline 30 & 6326.9789 & $+9 \pm 5$ & $+9 a$ & \\
\hline 41 & 6326.9893 & $-15 \pm 1$ & -15 & \\
\hline 224 & 6327.0019 & $-5 \pm 4$ & -5 & \\
\hline \multirow[t]{2}{*}{28} & 6326.9612 & $+3 \pm 10$ & +3 & \\
\hline & & Cluster Mean $=$ & $-2.3 \pm 1.6$ s.e. & \\
\hline
\end{tabular}

Rejected from mean as a likely non-member of cluster.

photometric data. The dereddening of the photometric data for cluster stars was therefore fairly straightforward, and was assisted by the existence of a spatial dependence of color excesses across the field.

Figure 3 is the variable-extinction diagram resulting from this analysis, with the derived color excesses being equivalent to that of a B0 star observed through the same amount of extinction. The luminosity estimates adopted for each star were obtained from either the star's MK type in Table 4 or the zero-age main-sequence (ZAMS) absolute magnitude corresponding to the star's intrinsic color (Turner 1979). There are no ambiguities in the derived color excesses for bright cluster stars, and the reddening of these stars correlates directly with the spatial location of the star. From the visibility of faint stars on the POSS survey for the NGC 1647 field, one can detect a number of elongated dust clouds running through the cluster. The densest portions of these tenuous clouds correspond to regions of high reddening for the colors of cluster stars, which makes it possible to map the clouds in some detail. Figure 4 illustrates the location of some of the more prominent dust lanes running through NGC 1647. Although none of these were detected in the survey by Lynds (1962), they all follow the general trend of Gould's Belt as it runs

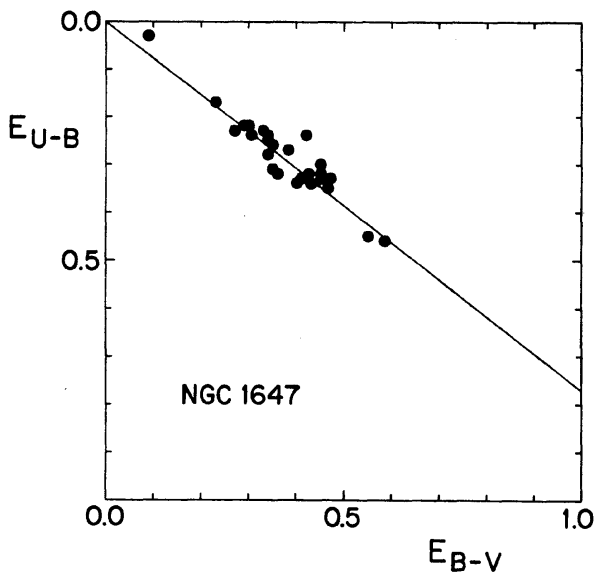

FIG. 1. Derived color excesses for NGC 1647 stars with well-established spectral types. The adopted reddening relation has a slope of $\mathrm{E}_{U-B} / \mathrm{E}_{B-V}=0.77$.

through this field. They clearly belong to the local dust system.

Field star contamination of open cluster fields usually increases in importance in direct proportion to the faintness and lateness of spectral type of the stars considered. Francic's (1989) proper motion survey of NGC 1647 is extremely useful for separating cluster members from foreground stars in the same line of sight. However, it appears to be less reliable at separating cluster members from background stars. There are three stars in Fig. 3 which have distance moduli indicating that they lie beyond NGC 1647, despite membership probabilities of $0.97-0.98$. All are pho-

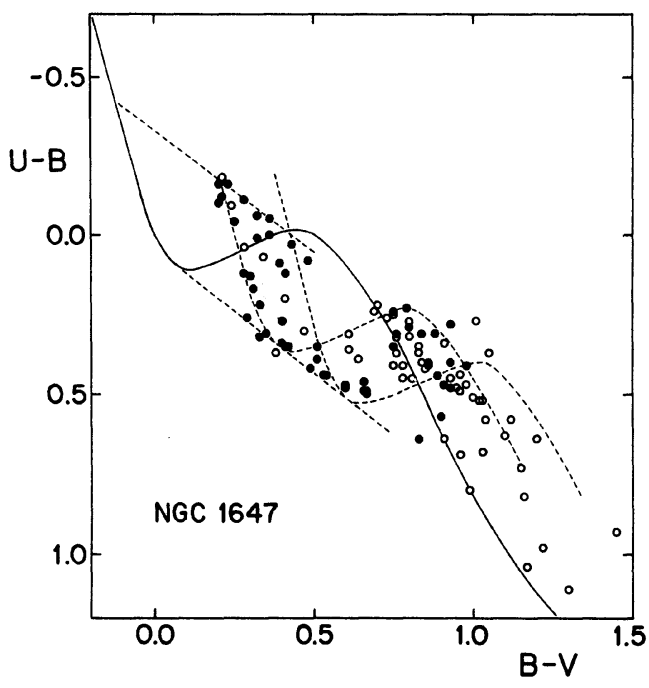

FIG. 2. Color-color diagram for NGC 129 stars. Photoelectric observations are plotted as dark circles, and photographic data as open circles. The continuous line is the intrinsic relation for main-sequence stars, while dashed lines represent the intrinsic relation reddened by $\mathrm{E}_{B-V}$ $=0.33$ and 0.55 , as well as reddening lines from $(B-V)_{0}$ $=-0.12$ (spectral type B7) and +0.07 (spectral type A2). 


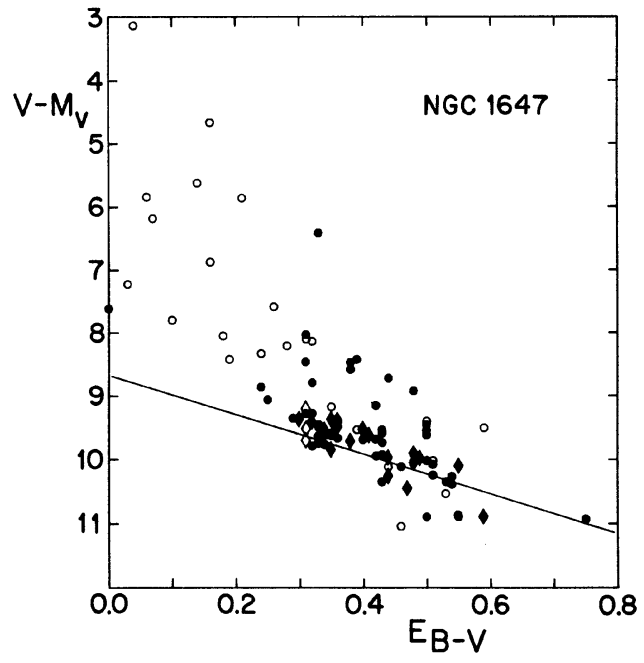

FIG. 3. Variable-extinction diagram for NGC 1647 stars based upon ZAMS-fitting (circles) and luminosities inferred from spectral types (diamonds). Filled symbols refer to stars with proper motion membership probabilities in excess of $50 \%$, while open symbols refer to stars with smaller probabilities or without proper motion membership data. The line of slope $R=3.09$ represents a lower envelope fit for likely ZAMS members of NGC 1647.

tometrically identified as F-type stars, which are relatively common field stars. We have assumed here that they are background stars.

The true run of apparent distance modulus with color excess for members of NGC 1647 in Fig. 3 should follow a relation similar in slope to the value of $R=A_{V} / \mathrm{E}_{B-V}$ $=2.86 \pm 0.30$ p.e. found by Turner (1976) in a previous variable-extinction study of this cluster. The spectroscopic distance moduli for bright cluster members do follow a relation of this slope, and it proves possible to use these objects as a guide for selecting a sample of 16 lower envelope points from the data for ZAMS-fitted cluster stars.

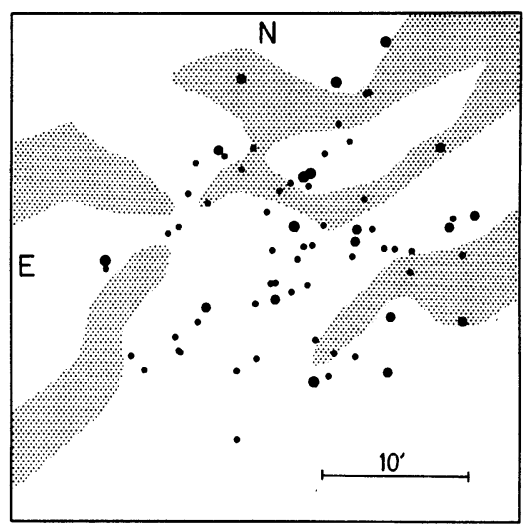

FIG. 4. Shaded regions denote areas of NGC 1647 for which the interstellar reddening is $\mathbf{E}_{B-V}=0.50$ or more. Circles represent the spatial location of adopted cluster members.
These points correspond to true, single, ZAMS members of NGC 1647, and their data yield a value of $R=3.00 \pm 0.12$ s.e. from regression and nonparametric straight line fits. The isolated point at $\mathrm{E}_{B-V}=0.75$ corresponds to CDS 217, which lies in the dust lane on the west side of the cluster. In order to avoid undue bias in the derived $R$ value, straight line fits were also made without this star. The result in this case was $R=3.09 \pm 0.16$ s.e., which was adopted as our final result. Most ZAMS-fitted cluster stars fall above this relation in Fig. 3 simply because they lie above the ZAMS in the cluster color-magnitude diagram.

\subsection{Cluster Stars}

Reddening-free data for likely members of NGC 1647 are summarized in Table 6 and plotted in Fig. 5. The ZAMS included in the figure corresponds to the mean distance modulus of $\left\langle V_{0}-M_{V}\right\rangle=8.67 \pm 0.02$ s.e. $( \pm 0.065$ s.d.) derived for the 16 stars identified in the previous section as defining the true ZAMS of NGC 1647. This corresponds to a cluster distance of $542 \pm 4 \mathrm{pc}$. The scatter of stars above the ZAMS could be due to several effects, including evolution (away from the ZAMS at the blue end and towards the ZAMS at the extreme red end), rotation, and duplicity. However, the available observational data are not sufficient to study this in any detail. The present study differs from previous photometric analyses of NGC 1647 primarily in making corrections for differential reddening across the field. The cluster color-magnitude diagram plotted in Fig. 5 is therefore much cleaner in appearance than any published previously. It is otherwise fairly typical of a cluster similar in age to the Pleiades cluster, with its main-sequence turnoff point defined by B7.5 stars. Based upon the observed properties of these stars, the estimated age for NGC 1647 is $1.1 \pm 0.1 \times 10^{8} \mathrm{yr}$ when referred to the evolutionary model/age calibration of Mermilliod (1981), and $1.9 \pm 0.4 \times 10^{8} \mathrm{yr}$ when referred to the more recent evolutionary models of Maeder \& Meynet (1988). By way of comparison, the period-age relation of Tammann (1970), which is tied to older evolutionary models, predicts an evolutionary age of about $8.6 \pm 1.7$ $\times 10^{7} \mathrm{yr}$ for a classical Cepheid with the period of SZ Tau.

The spatial distribution of likely cluster members is indicated in Fig. 4, which covers the same field as the NGC 1647 finder chart published in the U.S. Naval Observatory Catalogue (Hoag et al. 1961) and is centered on the same star. It seems clear from this diagram and from inspection of large-scale photographs of the field that the core region of NGC 1647 lies roughly 5'.5 northwest of the area usually considered to be the cluster center. This does not affect the arguments of Kovalenko (1968) that SZ Tau is projected against the cluster corona, although the Cepheid does lie very close to the extreme outer boundary of NGC 1647.

The cluster color-magnitude diagram of Fig. 5 is of interest with regard to the existence of gaps in cluster main-sequences (Böhm-Vitense \& Canterna 1974; Mermilliod 1976b). NGC 1647 presents no evidence for a significant gap at $(B-V)_{0}=0.25$, as observed in field stars and some open clusters by Böhm-Vitense \& Canterna (1974), 
TABLE 6. Reduced data for NGC 1647 stars.

\begin{tabular}{|c|c|c|c|c|c|c|c|c|c|c|c|}
\hline CDS & $(B-V)_{0}$ & $\mathrm{E}_{\mathrm{B}-\mathrm{V}^{\mathrm{a}}}$ & $\mathrm{V}_{\mathrm{o}}$ & CDS & $(B-V)_{0}$ & $\mathrm{E}_{\mathrm{B}-\mathrm{V}^{\mathrm{a}}}$ & $v_{o}$ & $\mathrm{CDS}$ & $(B-V)_{0}$ & $E_{B-V^{a}}$ & $\mathrm{~V}_{\mathrm{o}}$ \\
\hline SZ Tau & +0.56 & $0.31^{b}$ & 5.58 & 224 & +0.03 & 0.38 & 10.04 & 212 & +0.39 & 0.43 & 11.87 \\
\hline 29023 & -0.10 & 0.31 & 7.11 & 28 & +0.17 & 0.35 & 10.29 & 211 & +0.36 & 0.42 & 11.91 \\
\hline 29403 & -0.09 & 0.31 & 7.55 & 14 & -0.03 & 0.55 & 9.73 & 205 & +0.42 & 0.35 & 12.23 \\
\hline 15 & -0.11 & 0.48 & 7.12 & 33 & +0.06 & 0.33 & 10.45 & 217 & +0.19 & 0.75 & 11.04 \\
\hline 45 & -0.11 & 0.40 & 7.64 & 53 & +0.07 & 0.43 & 10.15 & 256 & +0.43 & 0.34 & 12.32 \\
\hline 22 & -0.09 & 0.30 & 8.15 & 9 & +0.12 & 0.50 & 10.05 & 95 & +0.48 & 0.33 & 12.53 \\
\hline 44 & -0.08 & 0.34 & 8.20 & 93 & +0.10 & 0.33 & 10.69 & 223 & +0.35 & 0.54 & 11.83 \\
\hline 102 & -0.08 & 0.41 & 8.07 & 46 & +0.13 & 0.39 & 10.51 & 214 & $+0.54:$ & 0.42 & 12.33 \\
\hline 3 & -0.11 & 0.44 & 8.26 & 35 & +0.09 & 0.33 & 10.72 & 215 & +0.46 & 0.48 & 12.36 \\
\hline 94 & -0.12 & 0.36 & 8.57 & 176 & +0.19 & 0.29 & 10.89 & 204 & +0.71 & 0.31 & 12.92 \\
\hline 42 & -0.02 & 0.36 & 8.58 & 97 & +0.03 & 0.51 & 10.21 & 222 & +0.66 & 0.32 & 12.96 \\
\hline 49 & -0.06 & 0.48 & 8.50 & 11 & +0.18 & 0.50 & 10.39 & 249 & +0.50 & $0.32^{\mathrm{b}}$ & 13.02 \\
\hline 51 & -0.08 & 0.48 & 8.54 & 351 & +0.12 & 0.50 & 10.61 & 173 & +0.57 & 0.33 & 13.09 \\
\hline 99 & -0.07 & 0.49 & 8.57 & 38 & +0.23 & 0.40 & 11.04 & 440 & +0.88 & 0.31 & 13.40 \\
\hline 54 & -0.10 & 0.47 & 8.64 & 47 & +0.27 & 0.50 & 10.85 & 353 & +0.73 & 0.24 & 13.64 \\
\hline 50 & -0.12 & 0.34 & 9.08 & 210 & +0.27 & 0.36 & 11.33 & 251 & +1.16 & $0.33^{b}$ & 13.40 \\
\hline 37 & -0.04 & 0.35 & 9.08 & 8 & +0.23 & 0.43 & 11.19 & 357 & $+0.53:$ & 0.51 & 12.85 \\
\hline 5 & -0.10 & 0.35 & 9.12 & 350 & +0.15 & 0.54 & 10.87 & 358 & +0.59 & 0.42 & 13.16 \\
\hline 10 & -0.10 & 0.59 & 8.42 & 96 & +0.16 & 0.53 & 11.02 & 201 & +0.68 & 0.32 & 13.55 \\
\hline 55 & -0.11 & 0.55 & 8.61 & 177 & +0.41 & 0.31 & 11.92 & 430 & +0.70 & 0.35 & 13.46 \\
\hline 31 & -0.07 & 0.36 & 9.24 & 32 & +0.38 & 0.33 & 11.87 & 360 & +0.73 & 0.25 & 13.81 \\
\hline 48 & -0.08 & 0.43 & 9.06 & 43 & +0.26 & 0.54 & 11.44 & 361 & +0.65 & 0.59 & 12.78 \\
\hline 29 & -0.04 & 0.36 & 9.58 & 202 & +0.29 & 0.51 & 11.54 & 207 & +0.73 & $0.35^{b}$ & 13.58 \\
\hline 4 & +0.04 & 0.32 & 9.93 & 237 & +0.32 & 0.46 & 11.72 & & & & \\
\hline 41 & -0.03 & 0.44 & 9.81 & $51 \mathrm{~s}$ & +0.33 & 0.44 & 11.84 & & & & \\
\hline
\end{tabular}

a Reddening equivalent to that of a B0 star.

b Reddening from nearby stars.

but there is a fairly prominent gap between $M_{V}=+0.0$ and +0.3 at $(B-V)_{0}=-0.10$ which matches the B7-B8 main-sequence gap observed for field stars and in several open clusters of this age group by Mermilliod (1976b). Our spectroscopic sample of cluster stars is not extensive enough to investigate the possibility that this gap is related

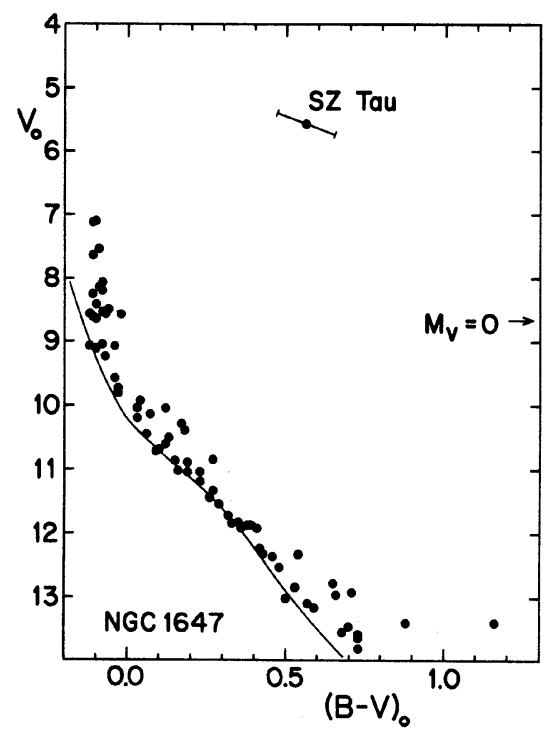

FIG. 5. Reddening-corrected color-magnitude diagram for likely members of NGC 1647. The extremes of variability for SZ Tau are indicated, while the continuous line represents the ZAMS for $V_{0}-M_{V}=8.67$. to stellar rotation, although, interestingly enough, most of the stars on the luminous side of the gap are rapidly rotating, as expected in this spectral interval (Fukuda 1982).

\subsection{SZ Tauri}

Since the radial velocity, proper motion, and inferred age of SZ Tau are an excellent match to the values found for members of NGC 1647, there is a strong case for cluster membership of the Cepheid. The location of SZ Tau on the periphery of the cluster corona makes the spatial coincidence with NGC 1647 less than ideal in many respects. However, one can also argue that this same property tends to improve the match in distance, since the depth of the cluster in the line of sight is smaller near the cluster periphery. The uncertainty in distance modulus of \pm 0.02 $( \pm 4 \mathrm{pc}$ ) found for members of the cluster core would need to be increased to \pm 0.08 for SZ Tau if the Cepheid were coincident with the nucleus of NGC 1647 , since the $\sim 19$ pc coronal radius of the cluster translates into a spread in distance modulus of this amount for a line of sight through the cluster center (spatial coincidence with the cluster nucleus does not necessarily imply location in the cluster nucleus). The depth of the cluster is much smaller at the periphery of the cluster corona, so it is reasonable to assume that an uncertainty in distance modulus of no more than \pm 0.02 applies to SZ Tau as well as to members of the cluster core.

The space reddening of SZ Tau derived by Turner (1984) can be revised according to the results of the present study. The two cluster members which bracket SZ Tau spatially are HD 29023 and HD 29403 (west and east 
of the Cepheid, respectively), and they have identical reddenings of $\mathrm{E}_{B-V}(\mathrm{~B} 0)=0.31$. There are no dust clouds visible in this part of NGC 1647, so it is reasonable to adopt an identical value for the reddening of SZ Tau. The color excess applicable to a star with the observed colors of the Cepheid is $\mathrm{E}_{B-V}=0.29 \pm 0.01$ (estimated uncertainty). A nearly identical value of $\mathrm{E}_{B-V}=0.31 \pm 0.03$ was obtained by Schmidt (1971) from spectroscopic measurements of the Cepheid at $\mathrm{H} \alpha$ and the $\mathrm{G}$ band. The corresponding luminosity for SZ Tau under the assumption that it is a member of NGC 1647 is $\left\langle M_{V}\right\rangle=-3.09 \pm 0.04$.

The luminosity of SZ Tau can also be derived from its radius, for which a few estimates have been made using the Baade-Wesselink (BW) method. Gieren (1985) derived a mean radius of $34.2 \pm 3.0 R_{\odot}$ for SZ Tau using the surface brightness variant of the BW method, and Moffett \& Barnes (1987b) obtained values of $38.94 \pm 3.24 R_{\odot}$ and $40.42 \pm 3.37 R_{\odot}$ in similar fashion using a fixed projection factor and period-dependent projection factor, respectively, for the conversion of the radial velocities to expansion velocities. The difference of $\sim 5 R_{\odot}$ in these estimates may relate to differences in the adopted observational data or in the treatment of interstellar reddening, to which the technique is sensitive. A radius for SZ Tau has also been obtained using the version of the BW method described by Turner (1988), in which the KHG spectral line index is used to isolate phases of identical effective temperature. This method is independent of the interstellar reddening for the Cepheid, but does introduce an empirical lineblocking and effective gravity factor for the $B$ and $V$ magnitudes which has many similarities to an identical factor introduced by Ivanov (1984) in his inverted version of the BW method. The value obtained for SZ Tau (Turner, unpublished) is $34.8 \pm 0.8 R_{\odot}$ (internal error estimate), which is in excellent agreement with Gieren's result.

Conversion of these radius estimates into luminosities requires a value for the mean effective temperature of SZ Tau, which can be established using the intrinsic color of $(\langle B\rangle-\langle V\rangle)_{0}=0.56 \pm 0.01$ derived from the Cepheid's space reddening. Various intrinsic color-effective temperature scales may be used. McWilliam's (1991) recent F supergiant calibration gives $T_{\text {eff }}=6062 \mathrm{~K}$ for this mean color, very close to what one obtains using the older calibration of Parsons (1971). The calibration of Teays \& Schmidt (1987) gives a smaller value of $T_{\text {eff }}=5906 \mathrm{~K}$, which is close to what one obtains using the model atmosphere calibration of Kurucz (1979). The luminosities corresponding to these values and a mean radius of $34.8 \pm 0.8$ $R_{\odot}$ for SZ Tau are $\left\langle M_{V}\right\rangle=-3.12 \pm 0.04$ (warm $T_{\text {eff }}$ scale) and $\left\langle M_{V}\right\rangle=-3.01 \pm 0.04$ (cool $T_{\text {eff }}$ scale), respectively, which are clearly an excellent match to the value found from ZAMS fitting. Very similar values are obtained using Gieren's (1985) radius estimate, while the radius estimates of Moffett \& Barnes (1987b) result in values which are 0m2-0m3 more luminous. Even if Moffett and Barnes' radius estimate for SZ Tau is not considered systematically too large, one can safely conclude that the luminosity of the Cepheid obtained from its likely membership in the

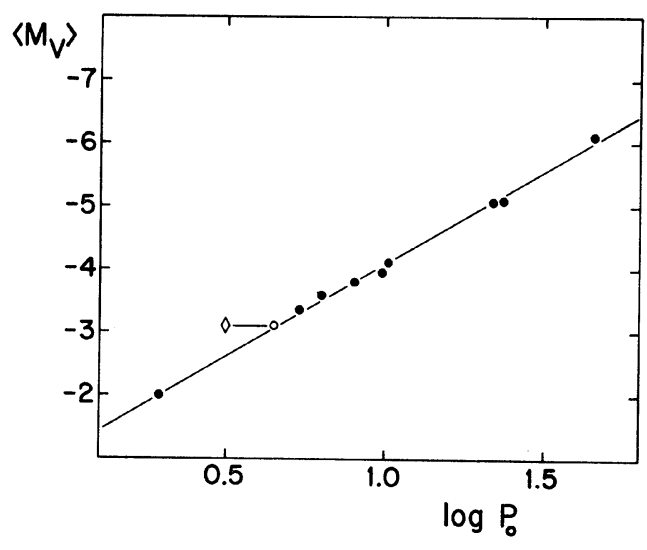

FIG. 6. Cepheid period-luminosity relation derived from members of open clusters and associations with distances based upon ZAMS fitting (dark circles). The data for SZ Tau as a member of NGC 1647 are plotted under the assumption that its variability is due to fundamental mode pulsation (open diamond) and first overtone pulsation (open circle), respectively. The fitted line is defined by $\left\langle M_{V}\right\rangle=-1.15-2.94 \log P_{0}$

corona of NGC 1647 is well supported by BW estimates for its radius, as argued previously by Gieren (1988).

The luminosity of SZ Tau derived from its cluster membership is plotted relative to the luminosities of other cluster/association Cepheids in Fig. 6, where the data for the Cepheids used for comparison purposes are summarized in Table 7. There are other calibrators available, but their luminosities have been obtained via spectroscopic and $\mathrm{H} \beta$ parallaxes for associated stars, or ZAMS fits based upon different principles than those used here, so they are not included. The tabulated data represent the best results currently available from an ongoing program to recalibrate Cepheid luminosities from cluster ZAMS fits. As such, they constitute an extremely homogeneous and internally self-consistent set of derived properties for cluster Cepheids, and one that can be usefully compared with existing calibrations. A least squares fit to the data of Fig. 6 yields a period-luminosity (PL) relation of the form $\left\langle M_{V}\right\rangle$ $=-1.15( \pm 0.06)-2.94( \pm 0.06) \log P_{0}$, with a rms de-

TABLE 7. Cepheid Iuminosities from cluster ZAMS fits.

\begin{tabular}{|c|c|c|c|c|}
\hline Cepheid & Cluster/Association & $\log P_{0}$ & $<\mathrm{Mv}>$ & Reference \\
\hline SU Cas & Cas R2 & 0.2899 & -1.99 & Turner \& Evans (1984) \\
\hline CV Mon & Anon (van den Bergh) & 0.7307 & -3.35 & Turner (1978) \\
\hline V367 Sct & NGC 6649 & 0.7989 & -3.58 & Tumer (1981) \\
\hline DL Cas & NGC 129 & 0.9031 & -3.80 & Turner, Forbes \& Pedreros (1992) \\
\hline S Nor & NGC 6087 & 0.9892 & -3.95 & Turner (1986) \\
\hline$\zeta \mathrm{Gem}$ & ADS $5742 \mathrm{AB}$ & 1.0065 & -4.11 & Tumer \& Fernie (1978) \\
\hline WZ Sgr & Anon (C1814-191a) & 1.3394 & -5.07 & Turner et al. (1992) \\
\hline SW Vel & Vel OB5 & 1.3700 & -5.09 & Tumer et al. (1992) \\
\hline SV Vul & Vul OB1 \& NGC 6834 & 1.6532 & -6.11 & Turner (1984) \\
\hline SZ Tau & NGC 1647 & $\begin{array}{l}0.4982^{\mathrm{a}} \\
0.6515^{\mathrm{b}}\end{array}$ & -3.09 & This Paper \\
\hline
\end{tabular}

a Assuming observed period results from fundamental mode pulsation.

b Assuming observed period results from first overtone pulsation. 
viation in $\left\langle M_{V}\right\rangle$ of \pm 0 m07. The corresponding rms deviations in the calibrations of Fernie \& McGonegal (1983) and Caldwell (1983) are $\pm 0 \mathrm{~m} 16$ and $\pm 0 \mathrm{~m} 135$, respectively, values twice as large as that obtained here. Clearly, sample homogeneity is an important factor for the galactic calibration of the Cepheid PL relation.

The luminosity of SZ Tau as a coronal member of NGC $1647,\left\langle M_{V}\right\rangle=-3.09 \pm 0.04$, is clearly overly luminous for the star's period of $3 \mathrm{~d} 148939$, which represents the best long term period fit to the $O-C$ data of Szabados (1977) and Trammell (1987). However, this discrepancy can easily be removed if it is assumed that the Cepheid is pulsating in the first overtone, rather than fundamental, mode. Known double-mode classical Cepheids of similar period have period ratios (first overtone/fundamental mode) of $P_{1} / P_{0} \sim 0.7$, and a more accurate value of $P_{1} / P_{0}=0.7025$ can be predicted for SZ Tau using the period ratio data summarized by Szabados (1988b). The corresponding pulsational period predicted for the unobserved fundamental mode of SZ Tau is $4 \mathrm{~d} 482059$, for which one predicts a luminosity of $\left\langle M_{V}\right\rangle=-3.07 \pm 0.07$ from the data for other calibrating Cepheids in Fig. 6. Clearly, the derived luminosity for SZ Tau as a cluster member $\left(\left\langle M_{V}\right\rangle=-3.09\right.$ $\pm 0.04)$ is, by itself, a strong argument for the identification of this Cepheid as an overtone pulsator.

The case for overtone pulsation is strengthened if one also considers the light curve of the variable. In recent years Fourier decomposition of Cepheid light curves has produced a variety of parameters which appear to be capable of indicating a star's pulsation mode (Simon 1988), and both $\varphi_{21}$ and $R_{21}$ for SZ Tau strongly suggest overtone pulsation (Antonello et al. 1990). SZ Tau is also recognized to be a member of the class of s-Cepheids based upon its low amplitude, sinusoidal, light curve. However, this classification does not itself imply overtone pulsation for the star, since the s-Cepheids do not as yet represent a completely homogeneous sample. For example, SU Cas also belongs to this class (Antonello et al. 1990), and yet its luminosity plotted in Fig. 6 appears to indicate fundamental mode pulsation for this star.

This point is addressed further in Fig. 7, which plots the $V$-band light curves of four Cepheids which seem to fit the GCVS characteristics of s-Cepheids-light amplitudes $\Delta V$ $<0$ m5 and $M-m=0.4-0.5$ (i.e., a duration for the rise from minimum to maximum light which is roughly $40 \%-$ $50 \%$ of the light cycle). The data plotted in Fig. 7 represent running averages over pulsational phase for the original observations, which are from Schmidt \& Gross (1990) for NO Cas and CN Tau, and from Milone (1970) for SZ Tau and SU Cas. These particular sources were chosen because they represent long, homogeneous, data sets with excellent phase coverage for the respective variables. From Fig. 7 it should be evident that NO Cas and SZ Tau have very similar sinusoidal light curves, with $M-m \approx 0.50$ and 0.49 , respectively, according to our estimates. By analogy with the light curve models of Stellingwerf et al. (1987) for overtone pulsation in RR Lyrae stars, both NO Cas and SZ Tau have light curve shapes typical of overtone pulsation. However, CN Tau and SU Cas have light curves more like

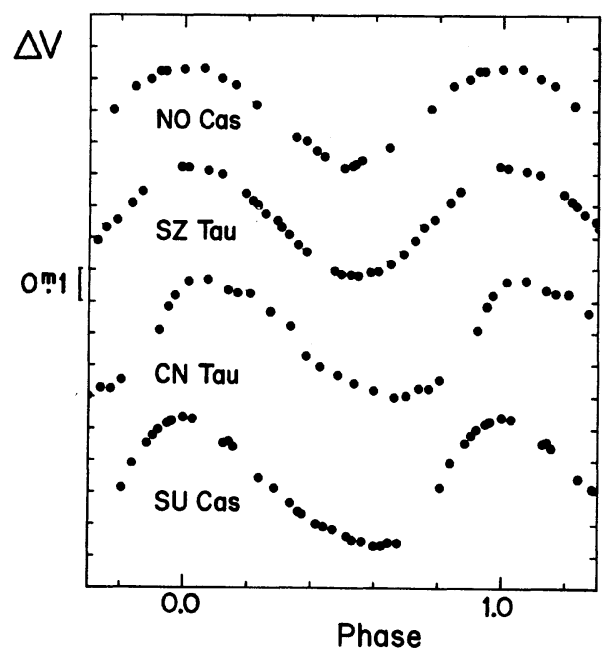

Fig. 7. Phase-smoothed light curves (to the same scale) for four short period pulsators identified as sCepheids. NO Cas and SZ Tau clearly belong to this class on the basis of light amplitude and light curve shape, but CN Tau and SU Cas have light curves skewed more like those of small amplitude fundamental mode Cepheids.

those of fundamental mode pulsators, with $M-m \approx 0.35$ and 0.40 , respectively. In fact, with these particular data sets, CN Tau and SU Cas do not strictly qualify as sCepheids. They seem likely to be low amplitude fundamental mode pulsators. Supporting evidence for fundamental mode pulsation in SU Cas will be presented in a separate study.

The evidence for overtone pulsation in SZ Tau does affect the arguments regarding its evolutionary age. The problem, however, lies in the published Cepheid periodage calibrations, which are based upon out-dated stellar evolutionary models and age estimates for clusters. If one uses the slope of the Cepheid period-age relation of Efremov (1977), normalized to the age estimates of Paper II for DL Cas in NGC 129, the inferred age for SZ Tau from $P_{0}=4$ d482059 becomes $\sim 1.5 \times 10^{8} \mathrm{yr}$, which agrees with the turnoff point age for NGC 1647. The likely match in age between SZ Tau and NGC 1647 is therefore preserved, and one can conclude that the Cepheid is almost certainly a cluster member.

\section{SUMMARY}

Based upon the observational data and the analysis presented in this study, the Cepheid SZ Tau is argued to be an outlying member of the open cluster NGC 1647. The spatial coincidence of the Cepheid with the cluster corona, as well as coincidences in radial velocity, proper motion, and age with cluster members, present a convincing case for cluster membership, which is also supported by independent estimates for the mean radius of SZ Tau. Moreover, the evidence presented by the derived luminosity of the Cepheid and its light curve strongly indicates that SZ Tau is pulsating in the first overtone, rather than fundamental, 
mode. The star may also be a spectroscopic binary (Szabados 1991), although there is no evidence for any contamination by the companion of the visible light from the system (Gieren 1985).

It is of interest to note that SZ Tau is not unique as an overtone pulsator located in a galactic cluster. The light curve parameters of EV Sct in NGC 6664, QZ Nor in NGC 6067 (cf. Coulson \& Caldwell 1985), and the binary Cepheid $\alpha$ UMi indicate that they are also likely overtone pulsators. When the double mode Cepheid V367 Sct in NGC 6649 is also considered, it should be evident that the presently-established selection of galactic clusters with member Cepheids constitutes a fairly broad sample which contains pulsating variables well distributed in both period (1d9-45:0) and pulsation mode. A detailed knowledge of the intrinsic properties of these cluster Cepheids should therefore provide a sound basis for the study of the PL relation for galactic Cepheids.

This investigation was supported by funds awarded through the Natural Sciences and Engineering Research
Council of Canada (NSERC), and was initiated during the author's tenure of an NSERC University Research Fellowship. I am grateful to the Directors of Kitt Peak National Observatory and the Dominion Astrophysical Observatory for generous allotments of observing time for this project. The assistance of the staff of the Dominion Astrophysical Observatory with the collection and reduction of the spectroscopic observations, as well as for the use of their facilities, is most gratefully acknowledged. I am endebted, in particular, to Graham Hill for his valuable advice and for permission to use his spectroscopic reduction programs. I am also endebted to Ron Lyons for measuring the imageintensifier spectra, Norm Scrimger for obtaining the 1984 spectra, Jim Burke for carefully assembling the published observational data for SZ Tau, Ed Schmidt for forwarding his observations of NO Cas and CN Tau, and Jean-Claude Mermilliod for providing a current listing of published papers on NGC 1647 and a cross reference list for previouslystudied stars. This research has made use of the SIMBAD database, operated at CDS, Strasbourg, France.

\section{REFERENCES}

Antonello, E., Poretti, E., \& Reduzzi, L. 1990, A\&A, 236, 138 Barnes, T. G., III, Moffett, T. J., \& Slovak, M. 1987, ApJS, 65, 307 Böhm-Vitense, E., \& Canterna, R. 1974, ApJ, 194, 629 Böhm-Vitense, E., \& Proffitt, C. 1985, ApJ, 296, 175

Buser, R. 1978, A\&A, 62, 411

Caldwell, J. A. R. 1983, Observatory, 103, 244

Caldwell, J. A. R., \& Coulson, I. M. 1987, AJ, 93, 1090

Coulson, I. M., \& Caldwell, J. A. R. 1985, MNRAS, 216, 671

Cuffey, J. 1937, Ann. Harvard Coll. Obs., 105, 403

Efremov, Y. N. 1964a, Peremennye Zvezdy, 15, 242

Efremov, Y. N. 1964b, Astron. Tsirk. No. 292, p. 3

Efremov, Y. N. 1977, Sov. Astr., 22, 161

Evans, N. R. 1985, in Cepheids: Theory and Observations, IAU Colloquium No. 82 , edited by B. F. Madore (Cambridge University Press, Cambridge), p. 79

Fernie, J. D. 1963, AJ, 68, 780

Fernie, J. D., \& McGonegal, R. 1983, ApJ, 275, 732

Francic, S. P. 1989, AJ, 98, 888

Fukuda, I. 1982, PASP, 94, 271

Gieren, W. P. 1985, A\&A, 148, 138

Gieren, W. P. 1988, PASP, 100, 262

Glushkova, E. V., \& Rastorguev, A. S. 1991, Sov. Astron. Lett., 17, 13

Hassan, S. M. 1972, MSAIt, 43, 279

Haynes, E. S. 1914, Lick Obs. Bull., 8, 85

Hill, G. 1982, Pub. DAO, 16, 59

Hill, G., \& Fisher, W. A. 1986, Pub. DAO, 16, 159

Hill, G., Fisher, W. A., \& Poeckert, R. 1982, Pub. DAO, 16, 43

Hoag, A. A., \& Applequist, N. L. 1965, ApJS, 12, 215

Hoag, A. A., Johnson, H. L., Iriarte, B., Mitchell, R. I., Hallam, K. L., \& Sharpless, S. 1961, Pub. U.S. Naval Obs., 17, 345

Ivanov, G. R. 1984, Ap\&SS, 105, 369

Johnson, H. L., \& Morgan, W. W. 1953, ApJ, 117, 313

Kovalenko, V. M. 1968, Astron. Tsirk. No. 473, p. 2

Kurucz, R. L. 1979, ApJS, 40, 1

Lynds, B. T. 1962, ApJS, 7, 1

Maeder, A., \& Meynet, G. 1988, A\&AS, 76, 411

McWilliam, A. 1991, AJ, 101, 1065

Mermilliod, J. C. 1976a, A\&AS, 24, 159

Mermilliod, J. C. 1976b, A\&A, 53, 289

Mermilliod, J. C. 1981, A\&A, 97, 235, 1981

Milone, E. F. 1970, IAU Inform. BulI. Variable Stars, No. 482
Moffett, T. J., \& Barnes, T. G., III 1987a, PASP, 99, 1206

Moffett, T. J., \& Barnes, T. G., III 1987b, ApJ, 323, 280

Parsons, S. B. 1971, MNRAS, 152, 121

Schaltenbrand, R., \& Tammann, G. A. 1971, A\&AS, 4, 265

Schmidt, E. G. 1971, AJ, 76, 1102

Schmidt, E. G. 1984, ApJS, 55, 455

Schmidt, E. G., \& Gross, B. A. 1990, PASP, 102, 978

Simon, N. R. 1988, in Pulsation and Mass Loss in Stars, edited by R. Stalio and L. A. Willson (Kluwer, Dordrecht), p. 27

Stellingwerf, R. F., Gautschy, A., \& Dickens, R. J. 1987, ApJ, 313, L75 Svolopoulos, S. N. 1962, ApJ, 13, 788

Szabados, L. 1977, Commun. Konkoly Obs. Hung. Acad. Sci., Budapest, No. 70

Szabados, L. 1988a, IAU Inform. Bull. Variable Stars, No. 3165

Szabados, L. 1988b, in Multimode Stellar Pulsations, edited by G. Kovacs, L. Szabados, and B. Szeidl (Konkoly Observatory, Budapest), p. 1

Szabados, L. 1991, Commun. Konkoly Obs. Hung. Acad. Sci., Budapest, No. 96

Tammann, G. A. 1970, in The Spiral Structure of Our Galaxy, IAU Symposium No. 38, edited by W. Becker and G. Contopoulos (Reidel, Dordrecht), p. 236

Teays, T. J., \& Schmidt, E. G. 1987, in Stellar Pulsation, edited by A. N. Cox, W. M. Sparks, and S. G. Starrfield (Springer, Berlin), p. 173

Trammell, S. R. 1987, JAAVSO, 16, 104

Turner, D. G. 1976, AJ, 81, 1125

Turner, D. G. 1978, JRASC, 72, 248

Turner, D. G. 1979, PASP, 91, 642

Turner, D. G. 1981, AJ, 86, 231

Turner, D. G. 1984, JRASC, 78, 229

Turner, D. G. 1986, AJ, 92, 111 (Paper I)

Turner, D. G. 1988, AJ, 96, 1565

Turner, D. G. 1989, AJ, 98, 2300

Turner, D. G., \& Evans, N. R. 1984, ApJ, 283, 254

Turner, D. G., \& Fernie, J. D. 1978, IAU Inform. Bull. Variable Stars, No. 1509

Turner, D. G., Forbes, D., \& Pedreros, M. 1992, AJ, 104, 1132 (Paper II)

Turner, D. G., van den Bergh, S., Younger, P. F., Danks, T. A., \& Forbes, D. 1993, ApJS (in press)

Zug, R. S. 1933, Lick Obs. Bull., 16, 119 